\title{
Optimal control problem for a generalized sixth order Cahn-Hilliard type equation with nonlinear diffusion
}

\section{Gongcao Shi ${ }^{1}$, Changchun Liu ${ }^{1 *}$ and Zhao Wang ${ }^{2}$}

\footnotetext{
"Correspondence: liucc@jlu.edu.cn 'Department of Mathematics, Jilin University, Changchun, 130012, China

Full list of author information is available at the end of the article
}

\begin{abstract}
In this paper, we study the initial-boundary-value problem for a generalized sixth order Cahn-Hilliard type equation, which describes the separation properties of oil-water mixtures when a substance enforcing the mixing of the phases is added. The optimal control under boundary condition is given and the existence of optimal solution is proved.
\end{abstract}

MSC: Primary 49J20; secondary 35K35; $35 \mathrm{~K} 55$

Keywords: sixth order Cahn-Hilliard; existence; optimal control

\section{Introduction}

We consider the equation

$$
u_{t}=D^{2}\left[\gamma D^{4} u-a(u) D^{2} u-\frac{a^{\prime}(u)}{2}|D u|^{2}+f(u)+k u_{t}-\gamma_{2} D^{2} u_{t}\right]
$$

in $\Omega \times(0, T)$, where $\Omega=(0,1), \gamma>0, k>0$, and $\gamma_{2}>0$ with the initial and boundary conditions

$$
\begin{aligned}
& u(x, 0)=u_{0}, \quad \text { in } \Omega, \\
& u(x, t)=D^{2} u(x, t)=D^{4} u(x, t)=0, \quad \text { on } \partial \Omega .
\end{aligned}
$$

The function $f(u)$ stands for the derivative of a potential $F(u)$ with $F(u), a(u)$ approximated, respectively, by a sixth and a second order polynomial

$$
\begin{aligned}
& F(u)=\int_{0}^{u} f(s) d s=(u+1)^{2}\left(u^{2}+h_{0}\right)(u-1)^{2}, \\
& a(u)=a_{2} u^{2}+a_{0},
\end{aligned}
$$

where $a_{2}>0$.

The free energy functional proposed by Gompper et al. [1-4] has the form

$$
\psi(u)=\int_{\Omega} \varphi(u, \nabla u, \Delta u) d x,
$$


with the density given by

$$
\varphi(u, \nabla u, \Delta u)=f(u)+\frac{1}{2} a(u)|\nabla u|^{2}+\frac{1}{2} \gamma(\Delta u)^{2} .
$$

Here $u$ is the scalar order parameter, which is proportional to the local difference between oil and water concentrations. The properties of the amphiphile and its concentration enter model (1.1) implicitly via (1.4) and (1.5). $F(u)$ has three minima at $u=-1, u=1$, and $u=0$, which describe the oil, water and disordered microemulsion phases. In [2-4], the coefficient $a(u)$ is approximated by the quadratic function (1.5) with constants $a_{0}$ of arbitrary sign and $a_{2}$ positive.

Like in the classical Cahn-Hilliard the theory the order parameter $u$ is a conserved quantity. Thus it satisfies the conservation law

$$
u_{t}+\nabla j=0
$$

with the mass flux $j$ given by the constitutive equation

$$
-j=\frac{\partial \mathcal{D}}{\partial \nabla \mu}=M \nabla \mu
$$

and $\mu$ representing the chemical potential

$$
\mu=\frac{\delta \psi}{\delta u}+\frac{\delta \mathcal{D}}{\delta u_{t}}
$$

where $\mathcal{D} \geq 0$, the dissipation potential, has the form

$$
\mathcal{D}\left(u_{t}, \nabla u_{t}, \nabla \mu\right)=\frac{1}{2} k\left(u_{t}\right)^{2}+\frac{1}{2} \gamma_{2}\left|\nabla u_{t}\right|^{2}+\frac{1}{2} M|\nabla \mu|^{2},
$$

and $M$ is the mobility, $k, \gamma_{2}$ are the viscosity coefficients corresponding to the rate of the order parameter and its spatial gradient.

The first variation $\frac{\delta \psi}{\delta u}$ is defined by the condition that

$$
\left.\frac{d}{d \lambda} \int_{\Omega} \psi(u+\lambda \zeta, \nabla u+\lambda \nabla \zeta, \Delta u+\lambda \triangle \zeta) d x\right|_{\lambda=0}=: \int_{\Omega} \frac{\delta \psi}{\delta u} \zeta d x
$$

most hold for all test functions $\zeta \in C_{0}^{\infty}(\Omega)$. In the case of free energy this leads to the following expressions:

$$
\begin{aligned}
& \frac{\delta \psi}{\delta u}=f(u)-a(u) \Delta u-\frac{a^{\prime}(u)}{2}|\nabla u|^{2}+\gamma \Delta^{2} u, \\
& \frac{\delta \mathcal{D}}{\delta u_{t}}=k u_{t}-\gamma_{2} \Delta u_{t} .
\end{aligned}
$$

From the above discussions we know that

$$
\mu=f(u)-a(u) \Delta u-\frac{a^{\prime}(u)}{2}|\nabla u|^{2}+\gamma \Delta^{2} u+k u_{t}-\gamma_{2} \Delta u_{t} .
$$


Combining (1.6)-(1.13) we get the following conserved evolution system:

$$
\begin{aligned}
& u_{t}-\nabla(M \nabla \mu)=0, \\
& \mu=f(u)-a(u) \Delta u-\frac{a^{\prime}(u)}{2}|\nabla u|^{2}+\gamma \Delta^{2} u+k u_{t}-\gamma_{2} \Delta u_{t},
\end{aligned}
$$

where $\Omega \subset \mathbb{R}^{3}$ is a bounded domain with the boundary $\partial \Omega$, occupied by the ternary mixture, and $(0, T)$ is the time interval. We endow this system with the initial and boundary condition (1.2) and (1.3), in this paper we consider the one-dimensional case with $M=1$.

Schimperna and Pawłow [5] studied (1.1) when $\gamma_{2}=0$ with logarithmic potential

$$
F(r)=(1-r) \log (1-r)+(1+r) \log (1+r)-\frac{\sigma}{2} r^{2}, \quad \sigma>0 .
$$

They investigated the behavior of the solutions to the sixth order system as the parameter $\gamma$ tended to 0 , the uniqueness and regularization properties of the solutions have been discussed.

Pawłow and Zajączkowski [6] proved that the problem (1.1)-(1.5) with $k=\gamma_{2}=0$ under consideration is well posed in the sense that it admits a unique global smooth solution which depends continuously on the initial datum.

In past decades, the optimal control of distributed parameter system had received much attention in the academic field. A wide spectrum of problems in applications can be solved by methods of optimal control, such as chemical engineering and vehicle dynamics. Modern optimal control theories and applied models are not only represented by ODEs, but also by PDEs. Kunisch and Volkwein solved open-loop and closed-loop optimal control problems for the Burgers equation [7], Armaou and Christofides studied the feedback control of Kuramto-Sivashing equation [8].

Recently, many authors studied the optimal control problem for the pseudo-parabolic equation, such as Tian et al. [9-11], Zhao and Liu [12].

In this paper, we consider the optimal control problem for the following equation:

$$
\begin{gathered}
\left(u-k D^{2} u+\gamma_{2} D^{4} u\right)_{t}-\frac{\gamma}{\gamma_{2}} D^{2}\left(u-k D^{2} u+\gamma_{2} D^{4} u\right)+\frac{\gamma}{\gamma_{2}} D^{2} u \\
+D^{2}\left(\left(a(u)-\frac{\gamma k}{\gamma_{2}}\right) D^{2} u+\frac{a^{\prime}(u)}{2}|D u|^{2}\right)=D^{2} f(u)+B^{*} \bar{\omega}
\end{gathered}
$$

with (1.2)-(1.5).

When $y=u-k D^{2} u+\gamma_{2} D^{4} u$, we take the distributed optimal control problem

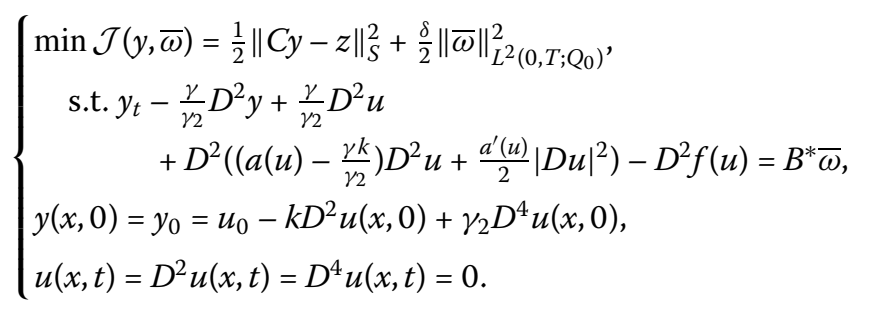

For fixed $T>0$, we set $\Omega=(0,1)$ and $Q=\Omega \times(0, T)$. Let $Q_{0} \subset Q$ be an open set with positive measure. 
Let $V=H_{0}^{1}(0,1), H=L^{2}(0,1) ; V^{*}=H^{-1}(0,1)$, and $H^{*}=L^{2}(0,1)$ are dual spaces, respectively, and we have

$$
V \hookrightarrow H=H^{*} \hookrightarrow V^{*}
$$

The extension operator $B^{*} \in L\left(L^{2}\left(0, T ; Q_{0}\right), L^{2}\left(0, T ; V^{*}\right)\right)$ is given by

$$
B^{*} q= \begin{cases}q, & q \in Q_{0}, \\ 0, & q \in Q / Q_{0} .\end{cases}
$$

The space $W(0, T ; V)$ is defined by

$$
W(0, T ; V)=\left\{y, y \in L^{2}(0, T ; V), y_{t} \in L^{2}\left(0, T ; V^{*}\right)\right\},
$$

which is a Hilbert space endowed with the common inner product.

The plan of the paper is as follows. In Section 2, we prove the existence of the weak solution in a special space. The optimal control is discussed in Section 3, and the existence of an optimal solution is proved.

\section{Existence of weak solution}

Consider the following the sixth order Cahn-Hilliard type equation:

$$
\begin{gathered}
\left(u-k D^{2} u+\gamma_{2} D^{4} u\right)_{t}-\frac{\gamma}{\gamma_{2}} D^{2}\left(u-k D^{2} u+\gamma_{2} D^{4} u\right)+\frac{\gamma}{\gamma_{2}} D^{2} u \\
+D^{2}\left(\left(a(u)-\frac{\gamma k}{\gamma_{2}}\right) D^{2} u+\frac{a^{\prime}(u)}{2}|D u|^{2}\right)=D^{2} f(u)+B^{*} \bar{\omega}
\end{gathered}
$$

under the initial value

$$
u(x, 0)=u_{0}
$$

and boundary condition

$$
u(x, t)=D^{2} u(x, t)=D^{4} u(x, t)=0,
$$

where $B^{*} \bar{\omega} \in L^{2}\left(0, T ; V^{*}\right)$ and the control item $\bar{\omega} \in L^{2}\left(0, T ; Q_{0}\right)$.

Let $y=u-k D^{2} u+\gamma_{2} D^{4} u$; the above problem is rewritten as

$$
\left\{\begin{array}{l}
y_{t}-\frac{\gamma}{\gamma_{2}} D^{2} y+\frac{\gamma}{\gamma_{2}} D^{2} u+D^{2}\left(\left(a(u)-\frac{\gamma k}{\gamma_{2}}\right) D^{2} u+\frac{a^{\prime}(u)}{2}|D u|^{2}\right)-D^{2} f(u)=B^{*} \bar{\omega}, \\
y(x, 0)=y_{0}=u_{0}-k D^{2} u_{0}+\gamma_{2} D^{4} u_{0}, \\
u(x, t)=D^{2} u(x, t)=D^{4} u(x, t)=0,
\end{array}\right.
$$

with (1.3)-(1.5).

Now, we give the definition of the weak solution to the problem (2.2) in the space $W(0, T ; V)$. 
Definition 2.1 A function $y(x, t) \in W(0, T ; V)$ is called a weak solution to problem (2.2), if

$$
\begin{aligned}
& \frac{d}{d t}(y, \phi)+\frac{\gamma}{\gamma_{2}}(D y, D \phi)-\frac{\gamma}{\gamma_{2}}(D u, D \phi) \\
& \quad-\left(D\left(a(u)-\frac{\gamma k}{\gamma_{2}}\right) D^{2} u+\frac{a^{\prime}(u)}{2}|D u|^{2}, D \phi\right)+(D f(u), D \phi)=\left(B^{*} \bar{\omega}, \phi\right)_{V^{*}, V}
\end{aligned}
$$

for all $\phi \in V$, a.e. $t \in[0, T]$ and $y_{0} \in H$ are valid.

Theorem 2.1 The problem (2.2) admits a weak solution $y(x, t) \in W(0, T ; V)$ in the interval $[0, T]$, if $B^{*} \bar{\omega} \in L^{2}\left(0, T ; V^{*}\right)$ and $y_{0} \in H$.

Proof Employ the standard Galerkin method.

The differential operator $A=-\partial_{x}^{2}$ is a linear unbounded self-adjoint operator in $H$ with $D(A)$ dense in $H$, where $H$ is a Hilbert space with a scalar product $(\cdot, \cdot)$ and norm $\|\cdot\|$.

There exists an orthogonal basis $\left\{\psi_{i}\right\}$ of $H$. Let $\left\{\psi_{i}\right\}_{i=1}^{\infty}$ be the eigenfunctions of the operator $A=-\partial_{x}^{2}$ with

$$
A \psi_{j}=\lambda_{j} \psi_{j}, \quad 0<\lambda_{1} \leq \lambda_{2} \leq \cdots, \text { as } j \rightarrow \infty
$$

For $n \in \mathbb{N}$, we define the discrete ansatz space by

$$
V_{n}=\operatorname{span}\left\{\psi_{1}, \psi_{2}, \ldots, \psi_{n}\right\} \subset V .
$$

Set $y_{n}(t)=y_{n}(x, t)=\sum_{i=1}^{n} y_{i}^{n}(t) \psi_{i}(x)$ and require $y_{n}(0, \cdot) \mapsto y_{0}$ in $H$ holds true.

To prove the existence of a unique weak solution to the problem (2.2), we are going to analyze the limiting behavior of sequences of smooth functions $\left\{y_{n}\right\}$ and $\left\{u_{n}\right\}$.

Performing the Galerkin procedure for the problem (2.2), we have

$$
\left\{\begin{array}{l}
y_{n, t}-\frac{\gamma}{\gamma_{2}} D^{2} y_{n}+\frac{\gamma}{\gamma_{2}} D^{2} u_{n} \\
\quad+D^{2}\left(\left(a\left(u_{n}\right)-\frac{\gamma k}{\gamma_{2}}\right) D^{2} u_{n}+\frac{a^{\prime}\left(u_{n}\right)}{2}\left|D u_{n}\right|^{2}\right)-D^{2} f\left(u_{n}\right)=B^{*} \bar{\omega} \\
y_{n}(x, 0)=y_{n, 0}=u_{n, 0}-k D^{2} u_{n}(x, 0)+\gamma_{2} D^{4} u_{n}(x, 0), \\
u_{n}(x, t)=D^{2} u_{n}(x, t)=D^{4} u_{n}(x, t)=0 .
\end{array}\right.
$$

According to ODE theory, there is a unique solution to (2.3) in the interval $\left[0, t_{n}\right]$. We should show that the solution is uniformly bounded when $t_{n} \rightarrow T$.

As a first step, multiplying the first equation of (2.3) by

$$
\mu_{n}=\gamma D^{4} u_{n}-a\left(u_{n}\right) D^{2} u_{n}-\frac{a^{\prime}\left(u_{n}\right)}{2}\left|D u_{n}\right|^{2}+f\left(u_{n}\right)+k u_{n, t}-\gamma_{2} D^{2} u_{n, t}
$$

and integrating with respect to $x$, we obtain

$$
\frac{d}{d t} E\left(u_{n}\right)+\left\|D \mu_{n}\right\|^{2}+k\left\|u_{n, t}\right\|^{2}+\gamma_{2}\left\|D u_{n, t}\right\|^{2}=\left(B^{*} \bar{\omega}, \mu_{n}\right)_{V^{*}, V}
$$


where

$$
E\left(u_{n}\right)=\int_{0}^{1}\left(\frac{\gamma}{2}\left|D^{2} u_{n}\right|^{2}+\frac{a\left(u_{n}\right)}{2}\left|D u_{n}\right|^{2}+F\left(u_{n}\right)\right) d x
$$

and

$$
F\left(u_{n}\right)=\left(u_{n}^{6}+\left(h_{0}-2\right) u_{n}^{4}+\left(1-2 h_{0}\right) u_{n}^{2}+h_{0}\right) .
$$

Applying a simple calculation, we have

$$
F\left(u_{n}\right) \geq C_{1} u_{n}^{6}-C_{0}
$$

where $C_{1}>0$ and $C_{0} \geq 0$.

Since $B^{*} \bar{\omega} \in L^{2}\left(0, T ; V^{*}\right)$ is a control item, we assume

$$
\left\|B^{*} \bar{\omega}\right\|_{V^{*}} \leq M
$$

Taking into account (2.4), (2.7), (2.8), (1.4), and integrating (2.4) with respect to time from 0 to $t$, we know

$$
\begin{aligned}
\int_{0}^{1}( & \left.\frac{\gamma}{2}\left|D^{2} u_{n}\right|^{2}+\frac{a_{2}}{2} u_{n}^{2}\left|D u_{n}\right|^{2}+C_{1} u_{n}^{6}\right) d x \\
& +\int_{0}^{t}\left\|D \mu_{n}\right\|^{2} d t+k \int_{0}^{t}\left\|u_{n, t}\right\|^{2} d t+\gamma_{2} \int_{0}^{t}\left\|D u_{n, t}\right\|^{2} d t \\
\leq & \int_{0}^{1} \frac{\left|a_{0}\right|}{2}\left|D u_{n}\right|^{2} d x+E\left(u_{n, 0}\right)+C_{0}+\int_{0}^{t}\left|\left(B^{*} \bar{\omega}, \mu_{n}\right)_{V^{*}, V}\right| d t \\
\leq & \varepsilon_{1} \int_{0}^{1} \frac{\left|a_{0}\right|}{2}\left|D^{2} u_{n}\right|^{2} d x+C\left(\varepsilon_{1}\right) \int_{0}^{1} u_{n}^{2} d x \\
& +E\left(u_{n, 0}\right)+C_{0}+\int_{0}^{t}\left\|B^{*} \bar{\omega}\right\|_{V^{*}}\left\|\mu_{n}\right\|_{V} d t \\
\leq & \varepsilon_{1} \int_{0}^{1} \frac{\left|a_{0}\right|}{2}\left|D^{2} u_{n}\right|^{2} d x+C\left(\varepsilon_{1}\right) \varepsilon_{2} \int_{0}^{1} u_{n}^{6} d x+C\left(\varepsilon_{2}\right) \\
& +E\left(u_{n, 0}\right)+C_{0}+C(\varepsilon) \int_{0}^{t}\left\|B^{*} \bar{\omega}\right\|_{V^{*}}^{2} d t+\varepsilon \int_{0}^{t}\left\|D^{2} \mu_{n}\right\|^{2} d t \\
= & \varepsilon_{1} \int_{0}^{1} \frac{\left|a_{0}\right|}{2}\left|D^{2} u_{n}\right|^{2} d x+C\left(\varepsilon_{1}\right) \varepsilon_{2} \int_{0}^{1} u_{n}^{6} d x+C\left(\varepsilon_{2}\right) \\
& +E\left(u_{n, 0}\right)+C_{0}+C(\varepsilon) \int_{0}^{t}\left\|B^{*} \bar{\omega}\right\|_{V^{*}}^{2} d t+\varepsilon \int_{0}^{t}\left\|u_{n, t}\right\|^{2} d t .
\end{aligned}
$$

Choosing $\varepsilon_{1}, \varepsilon_{2}$, and $\varepsilon$ sufficiently small, from the above inequality and the Poincaré inequality, we have

$$
\begin{aligned}
& \int_{0}^{1}\left|D^{2} u_{n}\right|^{2} d x \leq C, \\
& \int_{0}^{1}\left|D u_{n}\right|^{2} d x \leq C,
\end{aligned}
$$




$$
\begin{aligned}
& \int_{0}^{1} u_{n}^{6} d x \leq C \\
& \iint_{Q_{T}}\left|u_{n, t}\right|^{2} d x d t \leq C .
\end{aligned}
$$

From (2.11), we know

$$
\int_{0}^{1} u_{n}^{2} d x \leq C
$$

By virtue of (2.9), (2.10), and (2.13), we obtain

$$
\left\|u_{n}\right\|_{H^{2}} \leq C
$$

By Sobolev's imbedding theorem it follows from (2.14) that

$$
\left\|u_{n}\right\|_{L^{\infty}} \leq C, \quad\left\|D u_{n}\right\|_{L^{\infty}} \leq C .
$$

As a second step, multiplying (1.1) by $D^{2} u_{n}$ and integrating with respect to $x$, we obtain

$$
\begin{aligned}
\frac{1}{2} \frac{d}{d t} & \left(\int_{0}^{1}\left|D u_{n}\right|^{2} d x+k \int_{0}^{1}\left|D^{2} u_{n}\right|^{2} d x+\gamma_{2} \int_{0}^{1}\left|D^{3} u_{n}\right|^{2} d x\right)+\gamma \int_{0}^{1}\left|D^{4} u_{n}\right|^{2} d x \\
= & -\int_{0}^{1} D^{2} f\left(u_{n}\right) D^{2} u_{n} d x+\int_{0}^{1} a\left(u_{n}\right) D^{2} u_{n} D^{4} u_{n} d x \\
& +\int_{0}^{1} \frac{a^{\prime}\left(u_{n}\right)}{2}\left|D u_{n}\right|^{2} D^{4} u_{n} d x-\left(B^{*} \bar{\omega}, D^{2} u_{n}\right)_{V^{*}, V}
\end{aligned}
$$

From a simple calculation, we have

$$
\begin{aligned}
& a^{\prime}\left(u_{n}\right)=2 a_{2} u_{n}, \\
& D^{2} f\left(u_{n}\right)=f^{\prime}\left(u_{n}\right) D^{2} u_{n}+f^{\prime \prime}\left(u_{n}\right)\left(D u_{n}\right)^{2},
\end{aligned}
$$

where

$$
\begin{aligned}
& f^{\prime}\left(u_{n}\right)=\left(30 u_{n}^{4}+12\left(h_{0}-2\right) u_{n}^{2}+2\left(1-2 h_{0}\right)\right) \geq-C_{2}, \quad C_{2}>0, \\
& f^{\prime \prime}(u)=120 u_{n}^{3}+24\left(h_{0}-2\right) u_{n} .
\end{aligned}
$$

Thus it follows from (2.14), (2.18), and (2.19) that

$$
\begin{aligned}
\frac{1}{2} \frac{d}{d t} & \left(\int_{0}^{1}\left(D u_{n}\right)^{2} d x+k \int_{0}^{1}\left|D^{2} u_{n}\right|^{2} d x+\gamma_{2} \int_{0}^{1}\left|D^{3} u_{n}\right|^{2} d x\right)+\gamma \int_{0}^{1}\left|D^{4} u_{n}\right|^{2} d x \\
\leq & -\int_{0}^{1}\left(f^{\prime}\left(u_{n}\right) D^{2} u_{n}+f^{\prime \prime}\left(u_{n}\right)\left|D u_{n}\right|^{2}\right) D^{2} u_{n} d x \\
& +\int_{0}^{1}\left(a_{2} u_{n}^{2}+a_{0}\right) D^{2} u_{n} D^{4} u_{n} d x \\
& +\int_{0}^{1} \frac{a^{\prime}\left(u_{n}\right)}{2}\left|D u_{n}\right|^{2} D^{4} u_{n} d x+\left\|B^{*} \bar{\omega}\right\|_{V^{*}}\left\|D^{2} u_{n}\right\|_{V}
\end{aligned}
$$




$$
\begin{aligned}
\leq & C_{2} \int_{0}^{1}\left|D^{2} u_{n}\right|^{2} d x+C\left(\left\|u_{n}\right\|_{L^{\infty}}^{3}+\left\|u_{n}\right\|_{L^{\infty}}\right)\left\|D u_{n}\right\|_{L^{\infty}} \int_{0}^{1} D u_{n} D^{2} u_{n} d x \\
& +\left|a_{2}\right|\left\|u_{n}\right\|_{L^{\infty}}^{2} \int_{0}^{1} D^{2} u_{n} D^{4} u_{n} d x+\left|a_{0}\right| \int_{0}^{1} D^{2} u_{n} D^{4} u_{n} d x \\
& +\left|a_{2}\right| \int_{0}^{1} u_{n}\left|D u_{n}\right|^{2} D^{4} u_{n} d x+C(\varepsilon)\left\|B^{*} \bar{\omega}\right\|_{V^{*}}^{2}+\varepsilon \int_{0}^{1}\left|D^{4} u_{n}\right|^{2} d x \\
\leq & \frac{\gamma}{2} \int_{0}^{1}\left|D^{4} u_{n}\right|^{2} d x+C
\end{aligned}
$$

where $\varepsilon$ is sufficiently small.

By the Gronwall inequality, (2.21) implies

$$
\begin{aligned}
& \iint_{Q_{T}}\left|D^{4} u_{n}\right|^{2} d x d t \leq C, \\
& \int_{\Omega}\left|D^{3} u_{n}\right|^{2} d x \leq C .
\end{aligned}
$$

As a third step, multiplying (1.1) by $D^{4} u_{n}$ and integrating with respect to $x$, we obtain

$$
\begin{aligned}
\frac{d}{d t}( & \left.\frac{1}{2} \int_{\Omega}\left|D^{2} u_{n}\right|^{2} d x+\delta \int_{\Omega}\left|D^{3} u_{n}\right|^{2} d x+\gamma_{2} \int_{\Omega}\left|D^{4} u_{n}\right|^{2} d x\right)+\gamma \int_{\Omega}\left|D^{5} u_{n}\right|^{2} d x \\
= & -\int_{\Omega} f^{\prime}\left(u_{n}\right) D u_{n} D^{5} u_{n} d x \\
& -\int_{\Omega} D^{2}\left(a\left(u_{n}\right)\left|D^{2} u_{n}\right|\right) D^{4} u_{n} d x-\int_{\Omega} D^{2}\left(\frac{a^{\prime}\left(u_{n}\right)}{2}\left|D u_{n}\right|^{2}\right) D^{4} u_{n} d x \\
= & -\int_{\Omega} f^{\prime}\left(u_{n}\right) D u_{n} D^{5} u_{n} d x \\
& +\int_{\Omega} D\left(a\left(u_{n}\right)\left|D^{2} u_{n}\right|\right) D^{5} u_{n} d x+\int_{\Omega} D\left(\frac{a^{\prime}\left(u_{n}\right)}{2}\left|D u_{n}\right|^{2}\right) D^{5} u_{n} d x \\
= & I_{1}+I_{2}+I_{3} .
\end{aligned}
$$

On account of (2.15) and (2.10), we know

$$
I_{1} \leq C_{2}\left(C\left(\varepsilon_{3}\right) \int_{\Omega}\left|D u_{n}\right|^{2} d x+\varepsilon_{3} \int_{\Omega}\left|D^{5} u_{n}\right|^{2} d x\right) .
$$

On the other hand, by the Nirenberg inequality, we have

$$
\left\|D^{3} u_{n}\right\|_{\infty} \leq\left\|D^{5} u_{n}\right\|^{\frac{5}{8}}\left\|D u_{n}\right\|^{\frac{3}{8}}
$$

Hence, by the Hölder and Young inequalities, we obtain

$$
\begin{aligned}
\left|I_{2}\right| & =\left|\int_{\Omega} D\left(a_{2} u_{n}^{2}+a_{0}\right)\left(D^{2} u_{n}\right) D^{5} u_{n} d x\right| \\
& \leq 2 a_{2}\left\|u_{n}\right\|_{\infty}\left\|D u_{n}\right\|_{\infty}\left(\int_{\Omega}\left(D^{2} u_{n}\right)^{2} d x\right)^{\frac{1}{2}}\left(\int_{\Omega}\left|D^{5} u_{n}\right|^{2} d x\right)^{\frac{1}{2}}
\end{aligned}
$$




$$
\begin{aligned}
& +C\left\|D^{3} u\right\|_{\infty}\left\|u_{n}\right\|_{\infty}\left(\int_{\Omega} u_{n}^{2} d x\right)^{\frac{1}{2}}\left(\int_{\Omega}\left|D^{5} u_{n}\right|^{2} d x\right)^{\frac{1}{2}} \\
\leq & C\left(\varepsilon_{3}\right) C \int_{\Omega}\left|D^{5} u_{n}\right|^{2} d x .
\end{aligned}
$$

Similarly,

$$
\begin{aligned}
\left|I_{3}\right|= & \left|\int_{\Omega} D\left(\frac{a^{\prime}\left(u_{n}\right)}{2}\left|D u_{n}\right|^{2}\right) D^{5} u_{n} d x\right| \\
\leq & a_{2}\left\|D u_{n}\right\|_{\infty}^{2}\left(\int_{\Omega}\left|D u_{n}\right|^{2} d x\right)^{\frac{1}{2}}\left(\int_{\Omega}\left|D^{5} u_{n}\right|^{2} d x\right)^{\frac{1}{2}} \\
& +2 a_{2}\left\|u_{n}\right\|_{\infty}\left\|D u_{n}\right\|_{\infty}\left(\int_{\Omega}\left(D^{2} u_{n}\right)^{2} d x\right)^{\frac{1}{2}}\left(\int_{\Omega}\left|D^{5} u_{n}\right|^{2} d x\right)^{\frac{1}{2}} \\
\leq & C\left(\varepsilon_{3}\right) C \int_{\Omega}\left|D^{5} u_{n}\right|^{2} d x+C,
\end{aligned}
$$

the $\varepsilon_{3}$ is sufficiently small.

Therefore, by the Gronwall inequality, we have

$$
\begin{aligned}
& \sup _{0<t<T} \int_{\Omega}\left(D^{4} u_{n}\right)^{2} d x \leq C, \\
& \iint_{Q_{T}}\left|D^{5} u_{n}\right|^{2} d x d t \leq C .
\end{aligned}
$$

From a simple calculation, we have

$$
\begin{aligned}
\left\|y_{n}\right\|_{V} & =\left\|u_{n}-k D^{2} u_{n}+\gamma_{2} D^{4} u_{n}\right\|_{V}^{2} \\
& \leq C\left(\left\|u_{n}\right\|+\left\|D u_{n}\right\|+\left\|D^{2} u_{n}\right\|+\left\|D^{3} u_{n}\right\|+\left\|D^{4} u_{n}\right\|+\left\|D^{5} u_{n}\right\|\right) .
\end{aligned}
$$

From (2.14), (2.15), (2.26), and (2.27), we obtain

$$
\left\|y_{n}\right\|_{L^{2}(0, T ; V)} \leq C .
$$

As a fourth step, from (2.2), (2.14), (2.15), and the Sobolev embedding theorem, we have

$$
\begin{aligned}
\left\|y_{n, t}\right\|_{V^{*}} \leq & \left\|B^{*} \bar{\omega}\right\|_{V^{*}}+\left\|D^{5} u_{n}\right\|+\left\|D\left(\left(a\left(u_{n}\right)-\frac{\gamma k}{\gamma_{2}}\right) D^{2} u_{n}+\frac{a^{\prime}\left(u_{n}\right)}{2}\left|D u_{n}\right|^{2}\right)\right\| \\
& +\left\|D f\left(u_{n}\right)\right\| \\
\leq & \left\|B^{*} \bar{\omega}\right\|_{V^{*}}+\left\|D^{5} u_{n}\right\|+C\left\|u_{n}\right\|_{L^{\infty}}^{2}\left\|D^{3} u_{n}\right\|+C\left\|u_{n}\right\|_{L^{\infty}}\left\|D^{2} u_{n}\right\|^{2} \\
& +C\left\|u_{n}\right\|_{L^{\infty}}^{5}\left\|D u_{n}\right\|+C \\
\leq & C\left\|D^{5} u_{n}\right\|+C .
\end{aligned}
$$

Then

$$
\left\|y_{n, t}\right\|_{L^{2}\left(0, T ; V^{*}\right)} \leq C
$$


Thus, we have:

(i) For every $t \in[0, T]$, the sequence $\left\{y_{n}\right\}_{n \in \mathbb{N}}$ is bounded in $L^{2}(0, T ; H)$ as well as in $L^{2}(0, T ; V)$, which is independent of the dimension of the ansatz space $n$.

(ii) For every $t \in[0, T]$, the sequence $\left\{y_{n, t}\right\}_{n \in \mathbb{N}}$ is bounded in $L^{2}\left(0, T ; V^{*}\right)$, which is independent of the dimension of the ansatz space $n$.

Hence, we get $\left\{y_{n, t}\right\}_{n \in \mathbb{N}} \subset W(0, T ; V)$, and $\left\{y_{n, t}\right\}_{n \in \mathbb{N}}$ weak in $W(0, T ; V)$, weak star in $L^{\infty}(0, T ; H)$ and strong in $L^{2}(0, T ; H)$ to a function $y(x, t) \in W(0, T ; V)$. Obviously, the uniqueness of the solution is easy to obtain [13]. We omit it here.

To ensure that the norm of weak solution in the space $W(0, T ; V)$ can be controlled by the initial value and the control item, we need the following theorem.

Theorem 2.2 If $B^{*} \bar{\omega} \in L^{2}\left(0, T ; V^{*}\right)$ and $y_{0} \in H$, then there exist constants $C_{3}>0$ and $C_{4}>$ 0 , such that

$$
\|y\|_{W(0, T ; V)}^{2} \leq C_{3}\left(\left\|y_{0}\right\|_{H}^{2}+\|\bar{\omega}\|_{L^{2}\left(0, T ; Q_{0}\right)}^{2}\right)+C_{4} .
$$

Proof Similar to the proof of Theorem 2.1, we obtain

$$
\|u\| \leq C, \quad\|D u\| \leq C, \quad\|u\|_{V} \leq C, \quad\left\|D^{3} u\right\| \leq C .
$$

Multiplying the equation by $y$ and integrating the equation with respect to $x$, we obtain

$$
\begin{aligned}
\frac{1}{2} \frac{d}{d t} & \|y\|_{H}^{2}+\frac{\gamma}{\gamma_{2}}\|D y\|_{H}^{2} \\
= & \frac{\gamma}{\gamma_{2}} \int_{0}^{1} D y D u d x+\int_{0}^{1} D\left(\left(a(u)-\frac{\gamma k}{\gamma_{2}}\right) D^{2} u+\frac{a^{\prime}(u)}{2}|D u|^{2}\right) D y d x \\
& \quad-\int_{0}^{1} D y D f(u) d x+\left(B^{*} \bar{\omega}, y\right)_{V^{*}, V}
\end{aligned}
$$

From the Hölder and Young inequalities, we have

$$
\frac{\gamma}{\gamma_{2}} \int_{0}^{1} D y D u d x \leq C(\varepsilon)\|D u\|^{2}+\varepsilon\|D y\|^{2} .
$$

From (2.30), we have

$$
\begin{aligned}
\int_{0}^{1} D & \left(\left(a(u)-\frac{\gamma k}{\gamma_{2}}\right) D^{2} u+\frac{a^{\prime}(u)}{2}|D u|^{2}\right) D y d x \\
\leq & \left(\|u\|_{L^{\infty}}^{2}+C\right)\|D y\|\left\|D^{3} u\right\|+C\|u\|_{L^{\infty}}^{2}\|D u\|_{L^{\infty}}\left\|D^{2} u\right\|_{L^{\infty}}^{2}\|D y\| \\
& +\|D u\|^{3}\|D y\| \leq \varepsilon\|D y\|^{2}+C
\end{aligned}
$$

and

$$
-\int_{0}^{1} D y D f(u) d x \leq C_{2} \int_{0}^{1} D y d x \leq C\|D y\|+C \leq C .
$$


Note that

$$
\left(B^{*} \bar{\omega}, y\right)_{V^{*}, V} \leq\left\|B^{*} \bar{\omega}\right\|_{V^{*}}\|y\|_{V}
$$

From (2.31)-(2.34), we have

$$
\frac{1}{2} \frac{d}{d t}\|y\|_{H}^{2}+\frac{\gamma}{\gamma_{2}}\|D y\|_{H}^{2} \leq \varepsilon\|D y\|_{H}^{2}+C\left\|B^{*} \bar{\omega}\right\|_{V^{*}}^{2}+C .
$$

Integrating the above inequality with respect to $t$ yields

$$
\|y\|_{H}^{2} \leq\left\|y_{0}\right\|_{H}^{2}+C\left\|B^{*} \bar{\omega}\right\|_{L^{2}\left(0, T ; V^{*}\right)}^{2}+C
$$

By (2.36), (2.2), and (2.30), we deduce that

$$
\begin{aligned}
\left\|y_{t}\right\|_{V^{*}}^{2} \leq & \left\|B^{*} \bar{\omega}\right\|_{V^{*}}^{2}+\frac{\gamma}{\gamma_{2}}\|y\|_{V}^{2}+\frac{\gamma}{\gamma_{2}}\|D u\| \\
& +\left\|D\left(a(u) D^{2} u+\frac{a^{\prime}(u)}{2}|D u|^{2}\right)\right\|+\|D f(u)\| \\
\leq & \left\|B^{*} \bar{\omega}\right\|_{V^{*}}^{2}+C\|y\|_{V}^{2}+C \\
\leq & \left\|y_{0}\right\|_{H}^{2}+C\left\|B^{*} \bar{\omega}\right\|_{L^{2}\left(0, T ; V^{*}\right)}^{2}+C .
\end{aligned}
$$

From (2.36) and (2.37), we have

$$
\begin{aligned}
\|y\|_{W(0, T ; V)} & =\|y\|_{L^{2}(0, T ; V)}+\left\|y_{t}\right\|_{L^{2}\left(0, T ; V^{*}\right)} \\
& \leq C_{3}\left(\left\|y_{0}\right\|_{H}^{2}+\|\bar{\omega}\|_{L^{2}\left(0, T ; Q_{0}\right)}^{2}\right)+C_{4} .
\end{aligned}
$$

The proof is completed.

\section{Optimal problem}

In this section, we will study the distributed optimal control and the existence of the optimal solution is obtained based on Lions' theory.

We study the following problem when $\bar{\omega} \in L^{2}\left(0, T ; Q_{0}\right)$,

$$
\left\{\begin{array}{l}
\min \mathcal{J}(y, \bar{\omega})=\frac{1}{2}\|C y-z\|_{S}^{2}+\frac{\delta}{2}\|\bar{\omega}\|_{L^{2}\left(0, T ; Q_{0}\right)}^{2}, \\
\quad \text { s.t. } y_{t}-\frac{\gamma}{\gamma_{2}} D^{2} y+\frac{\gamma}{\gamma_{2}} D^{2} u+D^{2}\left(\left(a(u)-\frac{\gamma k}{\gamma_{2}}\right) D^{2} u+\frac{a^{\prime}(u)}{2}|D u|^{2}\right)-D^{2} f(u)=B^{*} \bar{\omega}, \\
y(x, 0)=y_{0}=u_{0}-k D^{2} u(x, 0)+\gamma_{2} D^{4} u, \\
u(x, t)=D^{2} u(x, t)=D^{4} u(x, t)=0,
\end{array}\right.
$$

where $y=u-k D^{2} u+\gamma_{2} D^{4} u$.

As we know that there exists a weak solution $y$ to (2.2), due to $u=\left(1-k \partial_{x}^{2}+\gamma_{2} \partial_{x}^{2}\right)^{-1} y$, we know that there exists a weak solution $u$ to (2.1). Let there be given an observation operator $C \in L(W(0, T ; V), S)$, in which $S$ is a real Hilbert space and $C$ is continuous.

We choose a performance index of tracking type

$$
\mathcal{J}(y, \bar{\omega})=\frac{1}{2}\|C y-z\|_{S}^{2}+\frac{\delta}{2}\|\bar{\omega}\|_{L^{2}\left(0, T ; Q_{0}\right)}^{2}
$$

where $z \in S$ is a desired state and $\delta>0$ is fixed. 
The optimal control problem as regards the further generalized sixth order CahnHilliard equation is

$$
\min \mathcal{J}(y, \bar{\omega})
$$

where $(y, \bar{\omega})$ satisfies the problem (2.2).

Let $X=W(0, T ; V) \times L^{2}\left(0, T ; Q_{0}\right)$ and $Y=L^{2}(0, T ; V) \times H$.

We define an operator $e=e\left(e_{1}, e_{2}\right): X \rightarrow Y$ by

$$
e(y, \bar{\omega})=e\left(e_{1}(y, \bar{\omega}), e_{2}(y, \bar{\omega})\right)
$$

where

$$
\left\{\begin{aligned}
e_{1}(y, \bar{\omega})= & \left(D^{2}\right)^{-1}\left(y_{t}-\frac{\gamma}{\gamma} D^{2} y+\frac{\gamma}{\gamma} D^{2} u\right. \\
& \left.+D^{2}\left(\left(a(u)-\frac{\gamma k}{\gamma_{2}}\right) D^{2} u+\frac{a^{\prime}(u)}{2}|D u|^{2}\right)-D^{2} f(u)-B^{*} \bar{\omega}\right), \\
e_{2}=y(x, 0)-y_{0} &
\end{aligned}\right.
$$

and $D^{2}$ is an operator from $H^{1}(0,1)$ to $H^{-1}(0,1)$.

Then (3.2) is rewritten as

$$
\min \mathcal{J}(y, \bar{\omega}) \quad \text { subject to } e=e(y, \bar{\omega})=0 .
$$

Now, we have the following theorem.

Theorem 3.1 There exists an optimal control solution to the problem.

Proof Let $(y, \bar{\omega}) \in X$ satisfy the equation $e=e(y, \bar{\omega})=0$. In view of (3.1), we have

$$
\mathcal{J}(y, \bar{\omega}) \geq \frac{\delta}{2}\|\bar{\omega}\|_{L^{2}\left(0, T ; Q_{0}\right)} .
$$

From Theorem 2.2, we have

$$
\|y\|_{W(0, T ; V)} \rightarrow \infty \quad \text { yields } \quad\|\bar{\omega}\|_{L^{2}\left(0, T ; Q_{0}\right)} \rightarrow \infty
$$

Hence

$$
\mathcal{J}(y, \bar{\omega}) \rightarrow+\infty, \quad \text { when }\|y, \bar{\omega}\|_{X} \rightarrow \infty
$$

As the norm is weakly lowered semi-continuous [14], we find that $\mathcal{J}$ is weakly lowered semi-continuous.

Since $\mathcal{J}(y, \bar{\omega}) \geq 0$ for all $(y, \bar{\omega}) \in X$ holds, there exists

$$
\eta=\inf \{\mathcal{J}(y, \bar{\omega}) \mid(y, \bar{\omega}) \in X \text { such that } e(y, \bar{\omega})=0\},
$$

which means that there exists a minimizing sequence $\left\{\left(y_{n}, \overline{\omega^{n}}\right)\right\}_{n \in \mathbb{N}}$ in $X$ such that

$$
\eta=\lim _{n \rightarrow \infty} \mathcal{J}\left(y_{n}, \bar{\omega}^{n}\right) \quad \text { and } \quad e=e\left(y_{n}, \bar{\omega}^{n}\right)=0, \quad \forall n \in \mathbb{N} .
$$


From (3.3), there exists an element $\left(y^{*}, \bar{\omega}^{*}\right) \in X$ such that

$$
\begin{aligned}
& y_{n} \rightarrow y^{*}, \quad y \in W(0, T ; V), \\
& \bar{\omega}^{n} \rightarrow \bar{\omega}^{*}, \quad \bar{\omega} \in L^{2}\left(0, T ; Q_{0}\right),
\end{aligned}
$$

when $n \rightarrow \infty$.

From (3.4), we have

$$
\lim _{n \rightarrow \infty} \int_{0}^{T}\left(y_{n}(t)-y^{*}(t), \phi(t)\right)_{V^{*}, V} d t=0, \quad \forall \phi \in L^{2}(0, T ; V) .
$$

Since $W(0, T ; V)$ is compactly embedded into $L^{2}\left(0, T ; L^{\infty}\right)$ and continuously embedded into $C(0, T ; H)$, we derive that $y_{n} \rightarrow y^{*}$ strongly in $L^{2}\left(0, T ; L^{\infty}\right)$ and $y_{n} \rightarrow y^{*}$ strongly in $C(0, T ; H)$, as $n \rightarrow \infty$. Then we also derive that $u_{n} \rightarrow u^{*}, D u_{n} \rightarrow D u^{*}, D^{2} u_{n} \rightarrow D^{2} u^{*}$, $D^{3} u_{n} \rightarrow D^{3} u^{*}, D^{4} u_{n} \rightarrow D^{4} u^{*}$ strongly in $C(0, T ; H)$, as $n \rightarrow \infty$.

As the sequence $\left\{y_{n}\right\}_{n \in \mathbb{N}}$ converges weakly, $\left\|y_{n}\right\|_{W(0, T ; V)}$ is bounded. Also, we see that $\left\|y_{n}\right\|_{L^{2}\left(0, T ; L^{\infty}\right)}$ is bounded based on the embedding theorem.

Since $y_{n} \rightarrow y^{*}$ strongly in $L^{2}\left(0, T ; L^{\infty}\right)$, we derive that $\left\|y^{*}\right\|_{L^{2}\left(0, T ; L^{\infty}\right)},\left\|u^{*}\right\|_{L^{2}\left(0, T ; L^{\infty}\right)}$, $\left\|D^{2} u^{*}\right\|_{L^{2}\left(0, T ; L^{\infty}\right)}$ and $\left\|D^{4} u^{*}\right\|_{L^{2}\left(0, T ; L^{\infty}\right)}$ are bounded.

Notice that

$$
\begin{aligned}
&\left|\int_{0}^{T} \int_{0}^{1}\left(D^{2} f\left(u_{n}\right)-D^{2} f\left(u^{*}\right)\right) \psi d x d t\right| \\
&=\left|\int_{0}^{T} \int_{0}^{1} D\left(f\left(u_{n}\right)-f\left(u^{*}\right)\right) D \psi d x d t\right| \\
& \leq C \mid \int_{0}^{T} \int_{0}^{1}\left(\left(u_{n}\right)^{4} D u_{n}+\left(u_{n}\right)^{2} D u_{n}+D u_{n}\right. \\
&\left.-\left(u^{*}\right)^{4} D u^{*}-\left(u^{*}\right)^{2} D u^{*}-D u^{*}\right) D \psi d x d t \mid \\
& \leq \int_{0}^{T}\left\|u_{n}\right\|_{L^{\infty}}^{4}\left\|D u_{n}-D u^{*}\right\|_{H}\|D \psi\|_{H} d t \\
&+\int_{0}^{T}\left\|\left(u_{n}\right)^{4}-\left(u^{*}\right)^{4}\right\|_{H}\left\|D u^{*}\right\|_{L^{\infty}}\|D \psi\|_{H} d t \\
&+\int_{0}^{T}\left\|\left(u_{n}\right)^{2}\right\|_{L^{\infty}}\left\|D u_{n}-D u^{*}\right\|_{H}\|D \psi\|_{H} d t \\
&+\int_{0}^{T}\left\|\left(u_{n}\right)^{2}-\left(u^{*}\right)^{2}\right\|_{H}\left\|D u^{*}\right\|_{L^{\infty}}\|D \psi\|_{H} d t \\
&+\int_{0}^{T}\left\|D u_{n}-D u^{*}\right\|_{H}\|D \psi\|_{H} d t \\
& \leq\left\|u_{n}\right\|_{C\left(0, T ; L^{\infty}\right)}^{4}\left\|D u_{n}-D u^{*}\right\|_{L^{2}(0, T ; H)}\|D \psi\|_{L^{2}(0, T ; H)} \\
&+\left(\left\|u_{n}\right\|_{C\left(0, T ; L^{\infty}\right)}^{3}+\left\|u^{*}\right\|_{C\left(0, T ; L^{\infty}\right)}^{3}\right)\left\|D u^{*}\right\|_{C\left(0, T ; L^{\infty}\right)}\left\|u_{n}-u^{*}\right\|_{L^{2}(0, T ; H)} \\
& \times\|D \psi\|_{L^{2}(0, T ; H)}+\left\|u_{n}\right\|_{C\left(0, T ; L^{\infty}\right)}^{2}\left\|D u_{n}-D u^{*}\right\|_{L^{2}(0, T ; H)}\|D \psi\|_{L^{2}(0, T ; H)} \\
&+\left(\left\|u_{n}\right\|_{C\left(0, T ; L^{\infty}\right)}+\left\|u^{*}\right\|_{C\left(0, T ; L^{\infty}\right)}\right)\left\|u_{n}-u^{*}\right\|_{L^{2}(0, T ; H)}\left\|D u^{*}\right\|_{C\left(0, T ; L^{\infty}\right)}
\end{aligned}
$$




$$
\begin{aligned}
& \times\|D \psi\|_{L^{2}(0, T ; H)}+\left\|D u_{n}-D u^{*}\right\|_{C(0, T ; H)}\|D \psi\|_{L^{2}(0, T ; H)} \\
\rightarrow & 0, \quad \forall \psi \in L^{2}(0, T ; V) .
\end{aligned}
$$

As we know

$$
\begin{aligned}
\mid \int_{0}^{T} & \int_{0}^{1}\left(D^{2}\left(a\left(u_{n}\right) D^{2} u_{n}+\frac{a^{\prime}\left(u_{n}\right)}{2}\left|D u_{n}\right|^{2}\right)\right. \\
& \left.-D^{2}\left(a\left(u^{*}\right) D^{2} u^{*}+\frac{a^{\prime}\left(u^{*}\right)}{2}\left|D u^{*}\right|^{2}\right)\right) \psi d x d t \mid \\
= & \mid \int_{0}^{T} \int_{0}^{1} D^{2}\left(a\left(u_{n}\right) D^{2} u_{n}-a\left(u^{*}\right) D^{2} u^{*}\right) \psi d x d t \\
& +\int_{0}^{T} \int_{0}^{1} D^{2}\left(\frac{a^{\prime}\left(u_{n}\right)}{2}\left|D u_{n}\right|^{2}-\frac{a^{\prime}\left(u^{*}\right)}{2}\left|D u^{*}\right|^{2}\right) \psi d x d t \mid \\
= & \left|I_{1}+I_{2}\right| .
\end{aligned}
$$

Note that

$$
\begin{aligned}
\left|I_{1}\right|= & \left|\int_{0}^{T} \int_{0}^{1} D^{2}\left(\left(a\left(u_{n}\right)-\frac{\gamma k}{\gamma_{2}}\right) D^{2} u_{n}-\left(a\left(u^{*}\right)-\frac{\gamma k}{\gamma_{2}}\right) D^{2} u^{*}\right) \psi d x d t\right| \\
\leq & \mid \int_{0}^{T} \int_{0}^{1} D\left(\left(a_{2}\left(u_{n}\right)^{2}+a_{0}-\frac{\gamma k}{\gamma_{2}}\right) D^{2} u_{n}\right. \\
& \left.-\left(a_{2}\left(u^{*}\right)^{2}+a_{0}-\frac{\gamma k}{\gamma_{2}}\right) D^{2} u^{*}\right) D \psi d x d t \mid \\
= & \mid \int_{0}^{T} \int_{0}^{1} D\left(a_{2}\left(u_{n}\right)^{2} D^{2} u_{n}-a_{2}\left(u^{*}\right)^{2} D^{2} u^{*}\right) D \psi d x d t \\
& +\int_{0}^{T} \int_{0}^{1} D\left(\left(a_{0}-\frac{\gamma k}{\gamma_{2}}\right) D^{2} u_{n}-\left(a_{0}-\frac{\gamma k}{\gamma_{2}}\right) D^{2} u^{*}\right) D \psi d x d t \mid \\
= & \left|I_{1}^{1}+I_{1}^{2}\right| .
\end{aligned}
$$

For $I_{1}^{1}$, we have

$$
\begin{aligned}
\left|I_{1}^{1}\right|= & \mid \int_{0}^{T} \int_{0}^{1}\left(2 a_{2} u_{n} D u_{n} D^{2} u_{n}-2 a_{2} u^{*} D u^{*} D^{2} u^{*}+a_{2}\left(u_{n}\right)^{2} D^{3} u_{n}\right. \\
& \left.-a_{2}\left(u^{*}\right)^{2} D^{3} u^{*}\right) D \psi d x d t \mid \\
\leq & 2\left|a_{2}\right| \int_{0}^{T}\left\|u_{n}\right\|_{L^{\infty}}\left\|D u_{n}\right\|_{L^{\infty}}\left\|D^{2} u_{n}-D^{2} u^{*}\right\|_{H}\|D \psi\|_{H} d t \\
& +2\left|a_{2}\right| \int_{0}^{T}\left\|u_{n}-u^{*}\right\|_{H}\left\|D u_{n}\right\|_{L^{\infty}}\left\|D^{2} u^{*}\right\|_{L^{\infty}}\|D \psi\|_{H} d t \\
& +2\left|a_{2}\right| \int_{0}^{T}\left\|u^{*}\right\|_{L^{\infty}}\left\|D u_{n}-D u^{*}\right\|_{H}\left\|D^{2} u^{*}\right\|_{L^{\infty}}\|D \psi\|_{H} d t \\
& +\left|a_{2}\right| \int_{0}^{T}\left(\left\|u_{n}\right\|_{L^{\infty}}+\left\|u^{*}\right\|_{L^{\infty}}\right)\left\|D^{3} u_{n}\right\|_{L^{\infty}}\left\|u_{n}-u^{*}\right\|_{H}\|D \psi\|_{H} d t
\end{aligned}
$$




$$
\begin{aligned}
& +\left|a_{2}\right| \int_{0}^{T}\left\|u^{*}\right\|_{L^{\infty}}^{2}\left\|D^{3} u_{n}-D^{3} u^{*}\right\|_{H}\|D \psi\|_{H} d t \\
\leq & 2\left|a_{2}\right|\left\|u_{n}\right\|_{C\left(0, T ; L^{\infty}\right)}\left\|D u_{n}\right\|_{C\left(0, T ; L^{\infty}\right)}\left\|D^{2} u_{n}-D^{2} u^{*}\right\|_{L^{(0, T ; H)}}\|D \psi\|_{L^{2}(0, T ; H)} \\
& +2\left|a_{2}\right|\left\|u_{n}-u^{*}\right\|_{L^{2}(0, T ; H)}\left\|D u_{n}\right\|_{C\left(0, T ; L^{\infty}\right)}\left\|D^{2} u^{*}\right\|_{C\left(0, T ; L^{\infty}\right)}\|D \psi\|_{L^{2}(0, T ; H)} \\
& +2\left|a_{2}\right|\left\|u^{*}\right\|_{C\left(0, T ; L^{\infty}\right)}\left\|D u_{n}-D u^{*}\right\|_{L^{2}(0, T ; H)}\left\|D^{2} u^{*}\right\|_{C\left(0, T ; L^{\infty}\right)}\|D \psi\|_{L^{2}(0, T ; H)} \\
& +\left|a_{2}\right|\left\|u_{n}\right\|_{C\left(0, T ; L^{\infty}\right)}\left\|D^{3} u_{n}\right\|_{L^{2}\left(0, T ; L^{\infty}\right)}\left\|u_{n}-u^{*}\right\|_{C(0, T ; H)}\|D \psi\|_{L^{2}(0, T ; H)} \\
& +\left\|u^{*}\right\|_{C\left(0, T ; L^{\infty}\right)}\left\|D^{3} u_{n}\right\|_{L^{2}\left(0, T ; L^{\infty}\right)}\left\|u_{n}-u^{*}\right\|_{C(0, T ; H)}\|D \psi\|_{L^{2}(0, T ; H)} \\
& +\left|a_{2}\right|\left\|u^{*}\right\|_{C\left(0, T ; L^{\infty}\right)}^{2}\left\|D^{3} u_{n}-D^{3} u^{*}\right\|_{L^{2}(0, T ; H)}\|D \psi\|_{L^{2}(0, T ; H)} \\
\rightarrow & 0, \quad \forall \psi \in L^{2}(0, T ; V) .
\end{aligned}
$$

Also we have

$$
\begin{aligned}
I_{1}^{2} & =\int_{0}^{T} \int_{0}^{1} D\left(\left(a_{0}-\frac{\gamma k}{\gamma_{2}}\right) D^{2} u_{n}-\left(a_{0}-\frac{\gamma k}{\gamma_{2}}\right) D^{2} u^{*}\right) D \psi d x d t \\
& \leq \int_{0}^{T}\left(a_{0}-\frac{\gamma k}{\gamma_{2}}\right)\left\|\left(D^{3} u_{n}-D^{3} u^{*}\right)\right\|_{H}\|D \psi\|_{H} d t \\
& \leq\left(a_{0}-\frac{\gamma k}{\gamma_{2}}\right)\left\|\left(D^{3} u_{n}-D^{3} u^{*}\right)\right\|_{C(0, T ; H)}\|D \psi\|_{L^{2}(0, T ; H)} \\
& \rightarrow 0, \quad \forall \psi \in L^{2}(0, T ; V) .
\end{aligned}
$$

Further, similar to (3.6), we have

$$
I_{2} \rightarrow 0, \quad \forall \psi \in L^{2}(0, T ; V) .
$$

From (3.5), we have

$$
\left|\int_{0}^{T} \int_{0}^{1}\left(B^{*} \bar{\omega}^{n}-B^{*} \bar{\omega}^{*}\right) \psi d x d t\right| \rightarrow 0, \quad \forall \psi \in L^{2}(0, T ; V)
$$

In view of the above discussion, we can conclude that

$$
e_{1}\left(y^{*}, \bar{\omega}^{*}\right)=0, \quad \forall n \in \mathbb{N} .
$$

Since $y^{*} \in W(0, T ; V)$, we have $y^{*}(0) \in H$. From $y_{n} \rightarrow y^{*}$ in $W(0, T ; V)$, we can infer that $y_{n}(0) \rightarrow y^{*}(0)$. Thus we obtain

$$
\left(y_{n}(0)-y^{*}(0), \psi\right) \rightarrow 0, \quad \forall \psi \in H
$$

which means that $e_{2}\left(y^{*}, \bar{\omega}^{*}\right)=0, \forall n \in \mathbb{N}$.

Hence, we can derive that $e\left(y^{*}, \bar{\omega}^{*}\right)=0, \forall n \in \mathbb{N}$.

In conclusion, there exists an optimal solution $\left(y^{*}, \bar{\omega}^{*}\right)$ to the problem. We can infer that there exists an optimal solution $\left(y^{*}, \bar{\omega}^{*}\right)$ to the viscous generalized Cahn-Hilliard equation due to $u=\left(1-k \partial_{x}^{2}+\gamma_{2} \partial_{x}^{4}\right)^{-1} y$. 


\section{Competing interests}

The authors declare that they have no competing interests.

\section{Authors' contributions}

All authors contributed equally to the manuscript and read and approved the final manuscript.

\section{Author details}

${ }^{1}$ Department of Mathematics, Jilin University, Changchun, 130012, China. ${ }^{2}$ School of Basic Science, Changchun

University of Technology, Changchun, 130012, China.

\section{Acknowledgements}

The authors would like to express their sincere thanks to the referee's valuable suggestions for the revision and improvement of the manuscript. This work is supported by the National Science Foundation of China (No. J1310022).

Received: 19 November 2014 Accepted: 24 March 2015 Published online: 03 April 2015

\section{References}

1. Gompper, G, Kraus, M: Ginzburg-Landau theory of ternary amphiphilic systems. I. Gaussian interface fluctuations. Phys. Rev. E 47, 4289-4300 (1993)

2. Gompper, G, Kraus, M: Ginzburg-Landau theory of ternary amphiphilic systems. II. Monte Carlo simulations. Phys. Rev. E 47, 4301-4312 (1993)

3. Gompper, G, Goos, J: Fluctuating interfaces in microemulsion and sponge phases. Phys. Rev. E 50, 1325-1335 (1994)

4. Gompper, G, Zschocke, S: Ginzburg-Landau theory of oil-water-surfactant mixtures. Phys. Rev. A 46, 4836-4851 (1992)

5. Schimperna, G, Pawłow, I: On a class of Cahn-Hilliard models with nonlinear diffusion. SIAM J. Math. Anal. 45, 31-63 (2013)

6. Pawłow, I, Zajaczkowski, W: A sixth order Cahn-Hilliard type equation arising in oil-water-surfactant mixtures. Commun. Pure Appl. Anal. 10, 1823-1847 (2011)

7. Kunisch, K, Volkwein, S: Control of the Burgers equation by a reduced-order approach using proper orthogonal decomposition. J. Optim. Theory Appl. 102, 345-371 (1999)

8. Armaou, A, Christofides, PD: Feedback control of the Kuramoto-Sivashinsky equation. Physica D 137, 49-61 (2000)

9. Shen, C, Tian, L, Gao, A: Optimal control of the viscous Dullin-Gottwald-Holm equation. Nonlinear Anal., Real World Appl. 11, 480-491 (2010)

10. Shen, C, Gao, A, Tian, L: Optimal control of the viscous generalized Camassa-Holm equation. Nonlinear Anal., Real World Appl. 11, 1835-1846 (2010)

11. Tian, L, Shen, C, Ding, D: Optimal control of the viscous Camassa-Holm equation. Nonlinear Anal., Real World Appl. 10 519-530 (2009)

12. Zhao, X, Liu, C: Optimal control problem for viscous Cahn-Hilliard equation. Nonlinear Anal. TMA 74, 6348-6357 (2011)

13. Hinze, M, Volkwein, S: Analysis of instantaneous control for the Burgers equation. Nonlinear Anal. TMA 50, 1-26 (2002)

14. Wouk, A: A Course of Applied Functional Analysis. Wiley-Interscience, New York (1979)

\section{Submit your manuscript to a SpringerOpen ${ }^{\ominus}$ journal and benefit from:}

- Convenient online submission

- Rigorous peer review

- Immediate publication on acceptance

- Open access: articles freely available online

- High visibility within the field

- Retaining the copyright to your article 